\title{
Efficacy prediction of targeted therapy for gastric cancer: The current status (Review)
}

\author{
WANJING FENG ${ }^{1,2}$ and XIAODONG $\mathrm{ZHU}^{1,2}$ \\ ${ }^{1}$ Department of Medical Oncology, Fudan University Shanghai Cancer Center; \\ ${ }^{2}$ Department of Oncology, Shanghai Medical College, Fudan University, Shanghai 200032, P.R. China
}

Received January 6, 2018; Accepted May 3, 2018

DOI: $10.3892 / \mathrm{mmr} .2018 .9145$

\begin{abstract}
Despite significant progress in the treatment of gastric cancer (GC), the prognosis remains poor and the mortality is high. Targeted drugs have been incorporated into routine treatment to improve treatment efficacy. However, the therapy response is still below 50\%. Therefore, there is a need to identify predictive factors for patient response to a specific drug in order to improve the efficacy of drug therapy. The present article reviewed the predictive factors for target therapy in GC, including epidermal growth factor receptor, human epidermal receptor 2, vascular endothelial growth factor family, molecules in the mesenchymal-epithelial transition pathway and the mammalian target of rapamycin. Additionally, the present review described the interactions between these molecules and signaling pathways.
\end{abstract}

\section{Contents}

1. Introduction

2. Anti-human epidermal growth factor receptor (EGFR) agents

3. Anti-HER2 agents

4. Anti-vascular endothelial growth factor (VEGF) or VEGF-receptor (VEGFR) agents

5. Anti-MET agents

6. Mammalian target of rapamycin (mTOR) inhibitors

7. Programmed cell death 1 (PD-1), programmed death-ligand (PD-L) 1 and cytotoxic T-lymphocyte-associated antigen 4 (CTLA-4) antibodies

8. Conclusion

Correspondence to: Dr Xiaodong Zhu, Department of Medical Oncology, Fudan University Shanghai Cancer Center, 270 Dong'An Road, Shanghai 200032, P.R. China

E-mail: xddr001@163.com

Key words: gastric cancer, predictive factor, targeted agent

\section{Introduction}

Gastric cancer (GC) is the fourth-most common malignant neoplasm and the second leading cause of cancer-associated death worldwide (1). Although surgery is considered the only curative method for GC, a large number of patients are unable to undergo surgery because of advanced disease stage at diagnosis (2-4). Accordingly, chemotherapy is one of the options for the treatment of advanced gastric cancer (AGC). However, the response rate of first-line chemotherapy regimens in GC is $\sim 40 \%$ In human epidermal receptor 2 (HER2)-positive patients, the response rate of first-line chemotherapy regimens using trastuzumab fails to reach $50 \%$ and the improvement of overall survival is limited (5). In patients who do not respond to chemotherapy, the benefit of chemotherapy is clearly limited, whereas the incidence of toxic reactions associated with the treatment is high (6). Therefore, it is important to identify patients who are sensitive to certain drugs. In addition, multidrug resistance (MDR) is an important contributor to drug non-response, and cellular resistance in AGC may be associated with the function of the MDR protein (7).

\section{Anti-human epidermal growth factor receptor (EGFR) agents}

Cetuximab and panitumumab are monoclonal antibodies against EGFR. Although several phase II clinical trials demonstrated the benefit of EGFR inhibitors in patients with $\mathrm{GC}(8,9)$, both randomized and open-label phase III trials of the drugs failed to prove the benefit of EGFR inhibitors in GC treatment. Cetuximab in Combination with Xeloda and Cisplatin in Advanced Esophago-gastric Cancer (EXPAND) clinical trial reported that cetuximab + cisplatin and capecitabine failed to improve the progression-free survival (PFS) (10). Furthermore, the Trial of Efficacy of Epirubicin, Oxaliplatin and Capecitabine (EOX) with/without Panitumumab in Previously Untreated Advanced Oesophagogastric Cancer (REAL3) showed that panitumumab + EOX did not increase the OS (11). Therefore, anti-EGFR treatments offer no survival benefits in non-selected patients with metastatic GC.

A previous study reported that wild-type KRAS proto-oncogene, GTPase (KRAS) could be a positive predictor of cetuximab efficacy in EGFR-positive gastric cancer cell lines (12) However, a prospective multi-center phase II trial 
assessed biomarkers in patients with gastroesophageal cancer treated with cetuximab + irinotecan, folinic acid and 5-FU (13). The study analyzed mutations of the KRAS (exons 12 and 13), B-Raf proto-oncogene, serine/threonine kinase (V600E), and phosphatidylinositol-4,5-bisphosphate 3-kinase catalytic subunit a (PIK3CA; exons 9 and 20) oncogenes, concluding that KRAS mutations were not suitable predictors of cetuximab efficacy (13).

Taken together, phase III clinical trials failed to confirm the efficacy of EGFR inhibitors in patients with GC and a phase II translational study did not identify any effective biomarkers for the prediction of cetuximab efficacy.

\section{Anti-HER2 agents}

Trastuzumab is a monoclonal antibody which binds to the extracellular domain of HER2 receptor (14). Trastuzumab is also the first approved targeted agent for the treatment of metastatic GC (5). HER2 is a promising predictive biomarker for the efficacy of trastuzumab efficacy (5).

In the Trastuzumab for GC (ToGA) phase III study, 594 GC/gastroesophageal junction cancer (GEJ), HER2 immunohistochemistry (IHC) 3+, or fluorescent in situ hybridization (FISH)-positive patients were randomly assigned to receive either chemotherapy (cisplatin combined with FU or capecitabine) + trastuzumab or chemotherapy alone (5). The OS and PFS significantly improved in patients treated with trastuzumab + chemotherapy. A post hoc analysis of OS by subgroups differentiated by protein expression level of HER2 showed the increased efficacy of trastuzumab associated with high expression of the protein (5). ToGA was a landmark study which utilized a targeted agent based on the measurement of a predictive biomarker, leading to a consensus about which patients may benefit from trastuzumab, the criteria for HER2-positive status being IHC3+ or IHC2+ and FISH+.

However, not all HER2-positive patients with GC benefit from trastuzumab. The efficacy prediction of trastuzumab has been investigated for years (15-19). In 2013, Gomez-Martin et al (16) reported that HER2 amplification, determined by the HER2/ centromeric probe for chromosome 17 (CEP17; 17p11.1-q11.1) ratio and HER2 copy number, demonstrated a significant positive correlation with sensitivity to HER2-targeted therapy and, therefore, could be considered a predictive factor of trastuzumab efficacy. Recently, Ock et al (17) reported a similar result to Gomez-Martin et al (16) indicating that HER2 gene amplification, identified using HER2/CEP17 ratio and HER 2 copy number, may be a positive predictive factor for treatment outcome of trastuzumab-based chemotherapy, especially in patients with HER 2 IHC $<2+$. However, another recent study evaluated the genomic alteration of HER2 using three different methods, including IHC, copy number variation and Ampliseq sequencing, and reported that the concomitant genomic alteration does not correlate with the treatment outcomes of HER2-targeted chemotherapy (18). In addition, Oyama et al (19) also tested the serum levels of HER2-extracellular domain (ECD) in patients with GC by chemiluminescent immunoassay and observed a correlation between HER2-ECD level and tissue HER2 status. The study also reported that alterations in the HER2-ECD level during chemotherapy positively correlated with therapy response in HER2-positive patients treated with trastuzumab-based chemotherapy. Accordingly, the researchers concluded that the serum HER2-ECD level could be a potential biomarker and monitoring marker for response to trastuzumab (19).

Lapatinib is a dual EGFR and HER2 inhibitor, the anticancer efficacy of which has been demonstrated in breast cancer (20), but not in GC. The Lapatinib Optimization Study in HER2 Positive GC (LOGiC) and Lapatinib Plus Paclitaxel Versus Paclitaxel Alone in the Second-Line Treatment of HER2-Amplified Advanced GC in Asian Populations (TyTAN) phase III trials were designed to evaluate the efficacy of lapatinib in patients with GC as first- and second-line treatments, respectively $(21,22)$. Both trials failed to achieve the primary endpoint, although the subgroup analysis of the LOGiC cohort showed a significant OS benefit in Asian patients and patients $<60$ years of age. Similarly, the efficacy of treatment with lapatinib + paclitaxel improved in IHC3+ patients compared with $\mathrm{IHC} 0 / 1+$ and $2+$ patients in the TyTAN trial $(21,22)$. Because of the disappointing results of the aforementioned lapatinib clinical trials, a number of studies have investigated the molecular mechanisms (23-26).

HER2-targeted therapy has served an important role in GC drug treatments for a number of years (27-29), however, there are challenges associated with both intrinsic and acquired drug resistance. In 2014, Eto et al (24) analyzed the over-expression and suppression of the microRNA-21 (miR-21)/phosphatase and tensin homolog (PTEN) pathway in GC cell lines revealing that over-expression of miR-21 led to decreased sensitivity of GC cells to trastuzumab, while the suppression of miR-21 restored the trastuzumab-resistance in GC cell lines. Recently, Zhang et al (25) reported that PTEN deficiency is a predictive factor for the early resistance to HER2-targeted therapy, including trastuzumab and lapatinib. In 2014, Hong et al (26) established a lapatinib-resistant OE19 subclone from HER2-positive lapatinib-sensitive cell lines, in order to analyze the function of lapatinib resistance. The authors identified a novel acquired mutation, SrcE527K, that activated both the phosphatidylinositide 3-kinase (PI3K) and mitogen-activated protein kinase (MAPK) pathways, and which may be a potential mechanism of lapatinib resistance, however, these findings require validation in primary tumor cells (26). Study by Hong et al (26) reported that the activation of the PI3K/protein kinase B (Akt) and MAPK pathways may be involved in HER2-targeted drug resistance.

The mesenchymal-epithelial transition (MET) pathway also serves a role in HER2-targeted drug resistance $(30,31)$. Kim et al (30) established a lapatinib-resistant SNU216 cell line with an epithelial-mesenchymal transition (EMT) phenotype. The researchers analyzed EMT-associated extracellular molecules and genes and identified testican-1, an extracellular molecule, the inhibition of which decreased testican-1-induced, MET-dependent downstream signaling and restored sensitivity to lapatinib (30). Therefore, this molecule may contribute to lapatinib resistance. A recent multicenter clinical trial conducted by De Silva et al (31) collected biopsy samples from patients with oesophagogastric adenocarcinoma (OGA) treated with neoadjuvant chemotherapy + lapatinib. The molecular analysis showed a significant positive correlation between MET activation and phosphor(p)-Erk level $(\mathrm{P}=0.0005)$ and $\mathrm{p}-\mathrm{PI} 3 \mathrm{~K} /$ total-PI3K ratio $(\mathrm{P}=0.0037)$, which 
indicated that MET contributed to lapatinib resistance, whereas neither insulin-like growth factor receptor (IGFR) nor receptor tyrosine-protein kinase erbB-3 were associated with lapatinib resistance (31).

\section{Anti-vascular endothelial growth factor (VEGF) or VEGF-receptor (VEGFR) agents}

The VEGF family is involved in the angiogenesis of malignant neoplasms (32). Bevacizumab is a humanized VEGF-A antibody that has been successful used for the treatment of renal (33), colorectal (34) and lung (35) cancer. However, two phase III clinical trials of GC patients failed to achieve the primary endpoint $(36,37)$. The Avastin in Gastric Cancer (AVAGAST) phase III study reported that bevacizumab + chemotherapy could be considered as a first-line treatment for patients with AGC, with improved PFS and tumor response rates, but not OS (36). The Bevacizumab plus Capecitabine and Cisplatin in Chinese Patients with Inoperable Locally Advanced or Metastatic Gastric or Gastroesophageal Junction Cancer (AVATAR) phase III study in China, with a design similar to that of the AVAGAST, also reported no difference in OS between the bevacizumab and placebo arms (37).

Based on the significant improvement in PFS in the AVAGAST trial, many oncologists believe it is important to identify patients who are sensitive to bevacizumab (38-41). In 2012, Yamashita-Kashima et al (38) reported that tumor VEGF levels and VEGF/basic fibroblast growth factor ratios were associated with bevacizumab sensitivity in vitro. Subsequently, Van Cutsem et al (39) hypothesized that angiogenic markers may be potential predictors for bevacizumab treatment outcome and conducted a study based on patients with AGC in the AVAGAST trial. They evaluated several angiogenic markers and found that plasma VEGF-A and neuropilin-1 were potential biomarkers for the prediction of treatment outcome in patients with AGC treated with chemotherapy + bevacizumab (39). In 2014, Han et al (40) analyzed bevacizumab pharmacokinetics in patients with AGC in the AVAGAST. The median clearance of bevacizumab was faster in patients with AGC compared with patients with other cancers ( 4.5 vs. $3 \mathrm{ml} / \mathrm{day} / \mathrm{kg}$ ), however, the mechanism of clearance remains unknown. The high clearance may contribute to the adverse treatment outcomes of bevacizumab (40). Recently, Hacker et al (41) evaluated angiopoietin-2 (Ang-2) expression in patients enrolled in the AVAGAS trial, reporting baseline plasma Ang-2 levels to be a prognostic biomarker for OS that was strongly positively associated with lymph node metastasis in AGC but not for bevacizumab efficacy. Therefore, although plasma VEGF-A and neuropilin-1 are potential biomarkers for bevacizumab treatment outcome, they remain to be validated (41).

Ramucirumab, a fully human monoclonal antibody against VEGFR-2, is the first drug approved by US Food and Drug Administration (FDA) for second-line treatment of AGC (42). A phase III clinical trial RAINBOW showed improved OS in the ramucirumab + paclitaxel arm compared with paclitaxel used as a single agent (43). Another phase III trial, REGARD, reported that ramucirumab as a single drug improved OS compared with that for the best supportive care [median OS: 5.2 months vs. 3.8 months, hazard ratio (HR) $0.776,95 \%$ CI
0.603-0.998; $\mathrm{P}=0.047]$ (44). However, the predictive factors of treatment efficacy are lacking for ramucirumab (45).

Apatinib, a VEGFR-2 tyrosine kinase inhibitor, was approved by Chinese Food and Drug Administration (CFDA) for patients with AGC refractory to $>2$ lines of prior chemotherapy. The phase III trial showed that OS and PFS time improved in the apatinib group compared with the placebo group (46).

\section{Anti-MET agents}

MET receptor, a tyrosine kinase receptor activated by hepatocyte growth factor (HGF) or scatter factor (SF), results in the activation of downstream pathways, including the PI3K/Akt and RAS-MAPK pathways (47).

Rilotumumab, an investigational monoclonal antibody, inhibits the MET signaling pathway (48). Although a phase II trial reported that rilotumumab could improve PFS and OS in MET-positive GC/GEJ patients (49), a phase III study, RELOMET1, failed to confirm the OS or PFS benefit from rilotumumab (50). Another phase III study also failed to prove the OS benefit of MET inhibitor onartuzumab administered in combination with mFolFox6 (fluorouracil, oxaliplatin and leucovorin) in MET-positive patients with GC (51). Foretinib, an inhibitor targeting both receptor tyrosine kinase (RTK) encoded by MET proto-oncogene (cMET) and VEGFR2, was effective in MET-positive patients with AGC in a 2013 phase II study (52).

The frequency of MET inhibitor responsiveness is low, the molecular mechanism of which has been analyzed. A 2015 study reported that short-form recepteur d'origine nantais (RON) pathways conferred intrinsic cMET-targeted therapy resistance in vivo and in vitro (53). Another recent study demonstrated that co-amplification of driver oncogenes, including HER 2 and MET, contributed to the acquired resistance to MET inhibitors (54). Ji et al (55) reported that high PI3K p110 $\alpha$ expression contributed to tyrosine kinase inhibitor resistance in GC xenografts. Musiani et al (56) reported that the induction of heat-shock protein 27 may limit the MET inhibitor efficacy in MET-positive patients with GC.

Furthermore, MET has cross-interactions with HER2 to modulate trastuzumab resistance (24), lapatinib resistance $(57,58)$ and HER2 expression (59). Several in vivo and in vitro experiments (24-26) have shown that drug resistance in HER2-targeted treatments may be associated with the MET pathway, which has been explained in detail in the anti-HER2 agents section of the present article. In 2014, a review by Sukawa et al (58) reported that HER2 over-expression was significantly positively correlated with p-Akt expression in GC tissues. Furthermore, p-Akt expression correlated with poor prognosis. These results suggest that the PI3K/Akt pathway serves a role in HER2-positive GC. Furthermore, PIK3CA mutations and/or PTEN inactivation may affect the effectiveness of HER2-targeted therapy (58).

The above studies indicated that efficacy of MET inhibitors has not been confirmed in phase III clinical trials and conclusive evidence of predictive factors remains lacking. However, the MET pathway could partially explain the resistance to HER2 inhibitors. 


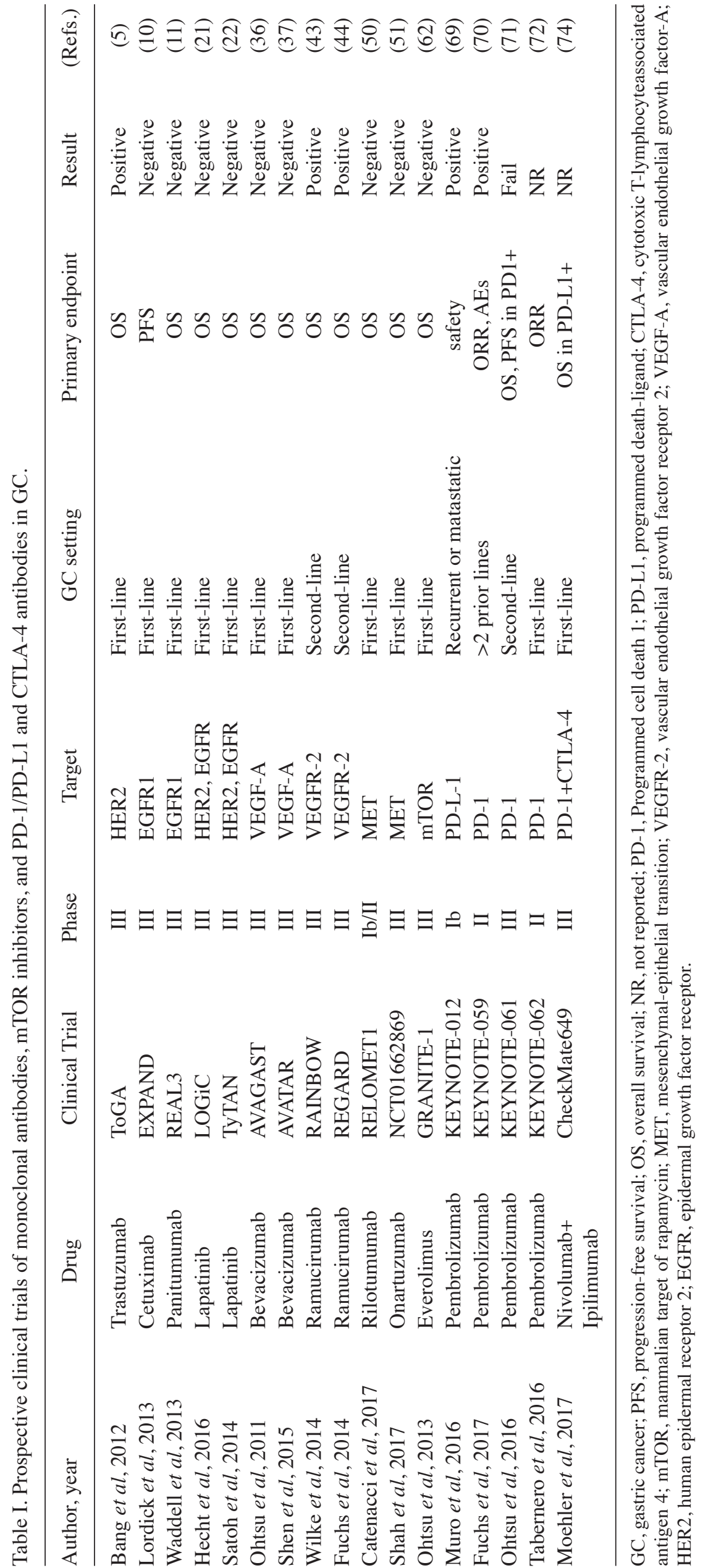




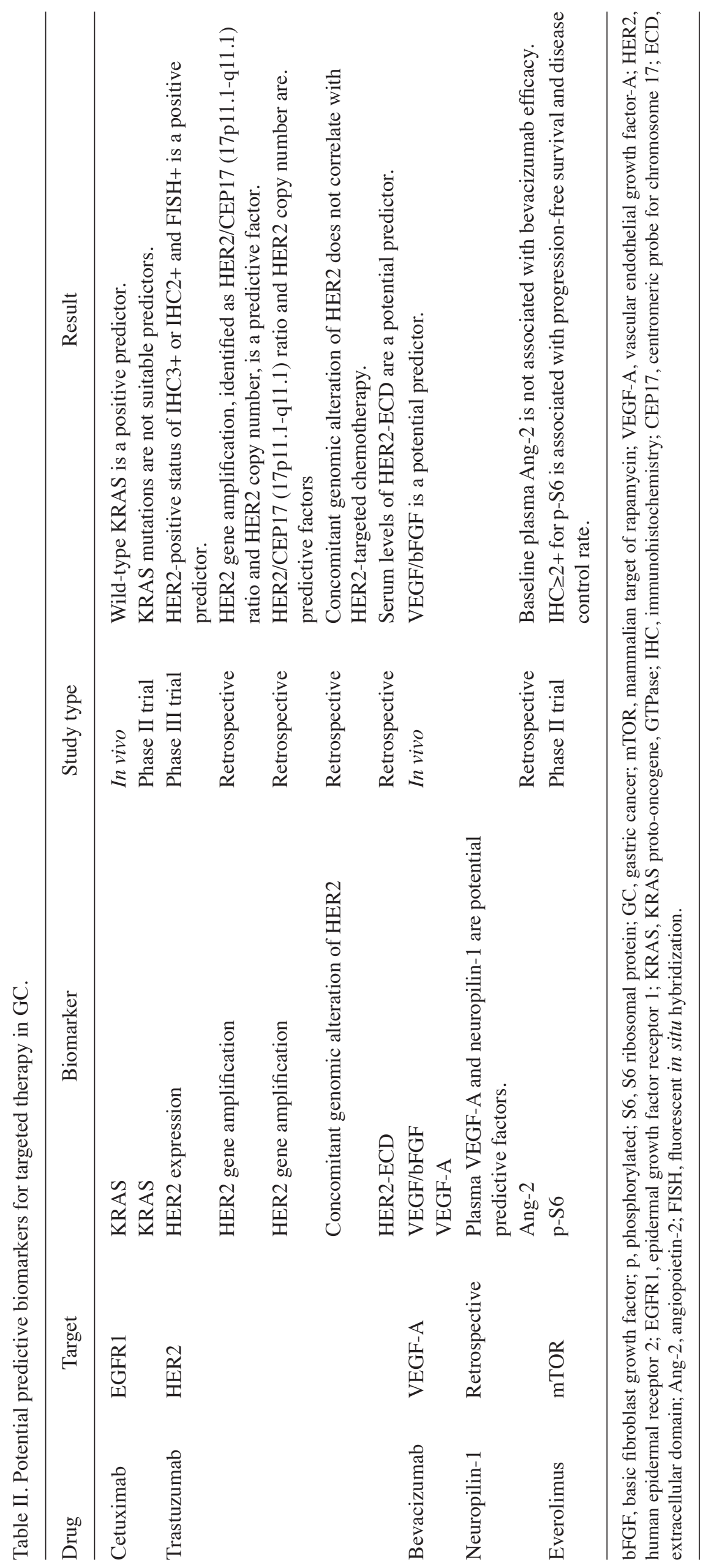




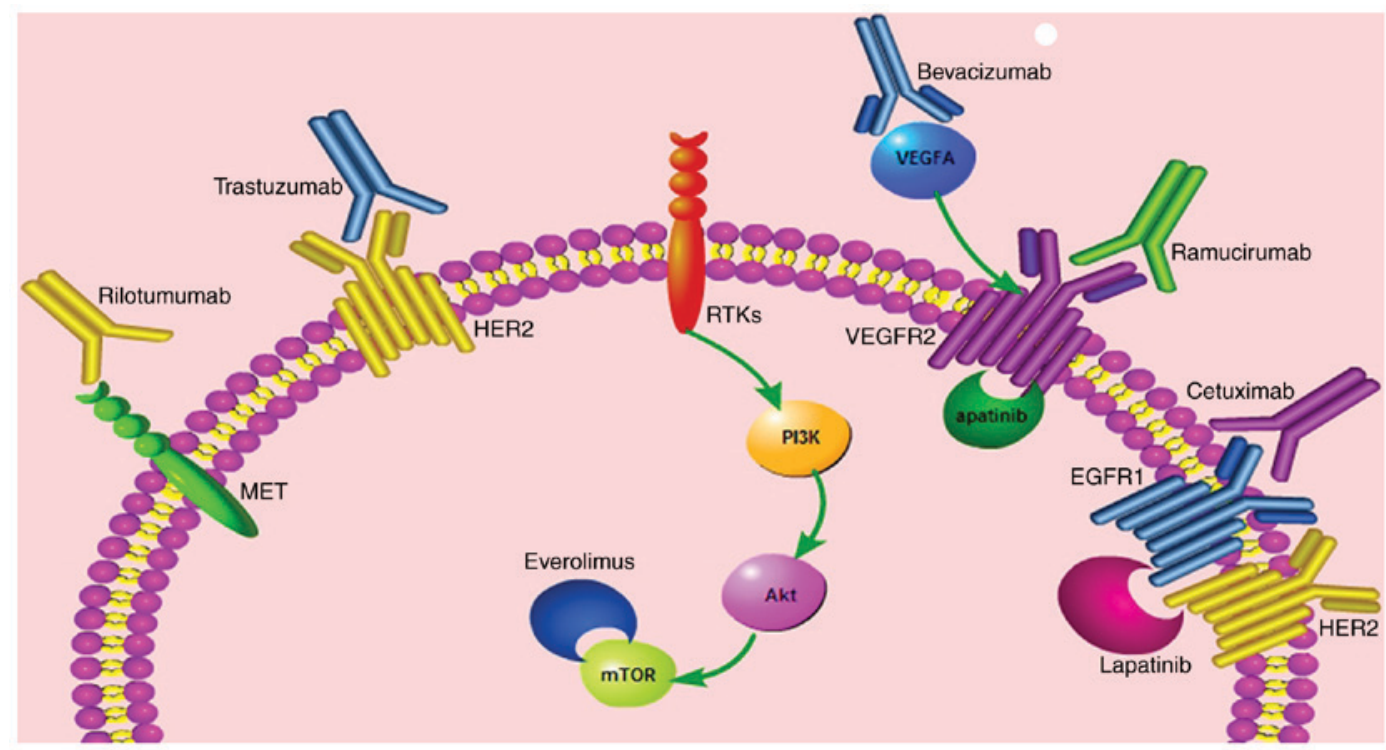

Figure 1. The mechanisms of targeted agents, including cetuximab, trastuzumab, lapatinib, bevacizumab, ramucirumab, apatinib, rilotumumab and everolimus. EGFR, epidermal growth factor receptor; HER2, human epidermal receptor 2; VEGFA, vascular endothelial growth factor A; MET, mesenchymal-epithelial transition; mTOR, mammalian target of rapamycin; RTK, receptor tyrosine kinase; PI3K, phosphoinositide 3-kinase; Akt, protein kinase B; VEGFR2, vascular endothelial growth factor receptor 2 .
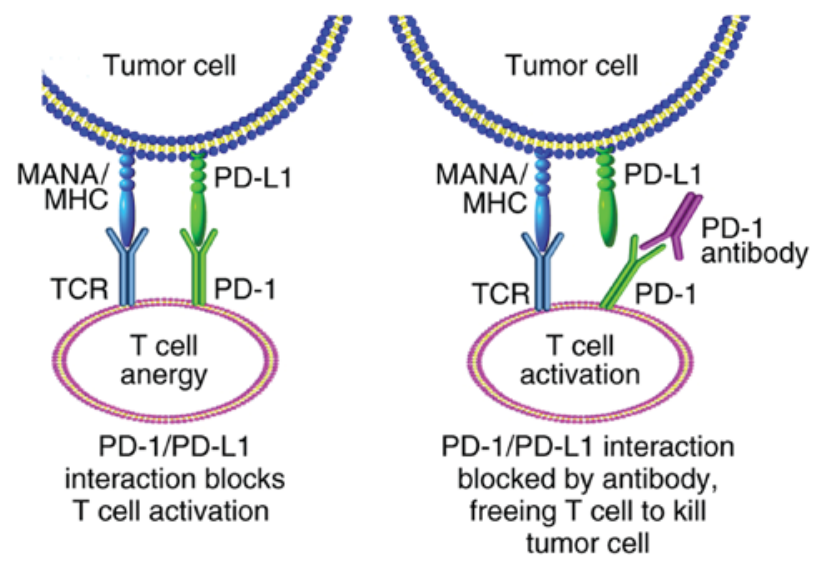

Figure 2. The mechanism of action of PD-1/PD-L1 agents. PD-1, programmed cell death 1; PD-L1, programmed death-ligand 1; MHC, major histocompatibility complex; MANA, mutant associated newborn antigen; TCR, T cell receptor.

\section{Mammalian target of rapamycin (mTOR) inhibitors}

mTOR, involved in the PI3K/Akt pathway, is a serine/threonine kinase that increases the production of proteins that stimulate cell growth, proliferation, angiogenesis and cell metabolism (60).

Everolimus is a mTOR inhibitor (61). Phase III study GRANITE-1 compared the effects of everolimus with the administration of placebo in patients with AGC with disease progression after one or two lines of chemotherapy and there was no significant difference in OS (62). To predict the efficacy of everolimus, Wainberg et al (63) conducted a phase II study, reporting a strong correlation between IHC staining $\geq 2+$ for $\mathrm{p}-\mathrm{S} 6$ ribosomal protein in tumor samples with improved PFS $(\mathrm{P}<0.0001)$ and disease control rate $(\mathrm{P}=0.0001)$ of patients, which indicated that $\mathrm{p}-\mathrm{S} 6$ ribosomal protein could be a potential biomarker.

Furthermore, mTOR inhibitors interacted with other target agents (64-66). Recently, Zhu et al (64) investigated the interaction between BEZ235, a dual PI3K/mTOR inhibitor, and trastuzumab in HER2-positive GC, demonstrating anticancer activity in Her2-positive GC and synergy with trastuzumab both in vivo and in vitro. The interaction between mTOR inhibitor everolimus and VEGFR inhibitors, including sunitinib (65) and vatalanib (66), reportedly improved anticancer activity, resulting in a significant reduction of tumor burden and long-term tumor control.

7.Programmed cell death 1 (PD-1), programmed death-ligand (PD-L) 1 and cytotoxic T-lymphocyte-associated antigen 4 (CTLA-4) antibodies

PD-1 is a negative costimulatory receptor expressed primarily on the surface of activated T cells (67). The binding of PD-1 to one of its ligands, PD-L1 or PD-L2, inhibits cytotoxic T-cell response (68). Phase Ib trial Keynote-012 has reported manageable toxicity and promising antitumor activity 
of pembrolizumab (pembro) in patients with AGC (69). Phase II Keynote-059 reported that pembro exhibited encouraging efficacy and manageable safety in patients with gastric/gastroesophageal junction cancer (G/GEJ) who had received $>2$ prior lines of therapy, although the survival and bio-marker data have yet to be published (70). According to Keynote-059, pembro has been approved by FDA for the treatment of patients with G/GEJ who had received $>2$ prior lines of therapy. Further phase III trials (Keynote-061 and 062) $(71,72)$ have yet to be published. On December 14th, Merck \& Co., Inc. (Whitehouse Station, NJ, USA) officially announced the failure of Keynote-061. The company announced that the clinical trial did not achieve the primary endpoint of OS and the difference between PFS was not statistically significant. The results of Keynote-061 and 062 have yet to be published. In addition to pembro, a phase III trial for another PD1-targeted drug, nivolumab, is also in progress and the result are not available.

Ipilimumab is an anti-CTLA-4 antibody, the anti-tumor mechanism of which is based on upregulation of antitumor immunity (73). A phase III clinical trial CheckMate649 aimed to evaluate ipilimumab + nivolumab (nivo) or nivo + chemotherapy vs. chemotherapy alone as first-line treatment for advanced G/GEJ, of which the results have not been reported (74).

The details of the clinical trials are summarized in Table I. The details of potential predictive bio-markers for targeted therapy are summarized in Table II. The mechanisms of targeted drugs are illustrated in Figs. 1 and 2.

\section{Conclusion}

The present article reviewed the efficacy predictors of targeted agents in GC. A total of four drugs have been approved by FDA and CFDA. Firstly, HER2 is a bio-marker for trastuzumab in patients with GC and, therefore, HER 2 analysis is recommended in patients with GC using standard test methods (HER2-positive status being IHC3+ or IHC2+ and FISH+) (5). HER2 amplification determined using HER2/CEP17 (17p11.1-q11.1) ratio and HER 2 copy number may be a predictor in HER2-targeted chemotherapy, which requires verification in further prospective studies $(16,17)$. In addition, the MET/PIK3CA/Akt pathway may be partially associated with HER 2 resistance $(24-26,30,31)$. Secondly, administration of ramucirumab, a VEGFR-2 antibody, is a standard therapy for second-line treatment of AGC $(43,44)$. However, the predictive factors of treatment efficacy are lacking for ramucirumab. Thirdly, apatinib, a VEGFR-2 tyrosine kinase inhibitor, was approved by CFDA for the treatment of patients with AGC refractory to $\geq 2$ lines of prior chemotherapy (46). Fourthly, pembro, a PD-1 antibody, has been approved by FDA in patients with G/GEJ who had received $>2$ prior lines of therapy (70). Lastly, the results of phase III studies suggest a modest and limited response to both cetuximab and bevacizumab in patients with GC $(10,36,37)$, however, efficacy predictions are currently lacking.

\section{Acknowledgements}

Not applicable.

\section{Funding}

This study was funded by The National Key Research and Development Program of China ( grant no. 2017YFC1308900).

\section{Availability of data and materials}

All data generated or analyzed during this study are included in this published article.

\section{Authors' contributions}

FW performed the literature search and wrote the article. ZX designed the review and drafted and revised the article.

\section{Ethics approval and consent to participate}

No applicable.

\section{Consent for publication}

No applicable.

\section{Competing interests}

The authors declare that they have no competing interests.

\section{References}

1. Siegel RL, Miller KD and Jemal A: Cancer statistics, 2016. CA Cancer J Clin 66: 7-30, 2016.

2. Ajani JA, Mayer RJ, Ota DM, Steele GD, Evans D, Roh M, Sugarbaker DJ, Dumas P, Gray C, Vena DA, et al: Preoperative and postoperative combination chemotherapy for potentially resectable gastric carcinoma. J Natl Cancer Inst 85: 1839-1844, 1993.

3. Leichman L, Silberman H, Leichman CG, Spears CP, Ray M, Muggia FM, Kiyabu M, Radin R, Laine L, Stain S, et al: Preoperative systemic chemotherapy followed by adjuvant postoperative intraperitoneal therapy for gastric cancer: A University of Southern California pilot program. J Clin Oncol 10: 1933-1942, 1992.

4. Chau I, Norman AR, Cunningham D, Waters JS, Oates J and Ross PJ: Multivariate prognostic factor analysis in locally advanced and metastatic esophago-gastric cancer-pooled analysis from three multicenter, randomized, controlled trials using individual patient data. J Clin Oncol 22: 2395-2403, 2004.

5. Bang YJ, Van Cutsem E, Feyereislova A, Chung HC, Shen L, Sawaki A, Lordick F, Ohtsu A, Omuro Y, Satoh T, et al: Trastuzumab in combination with chemotherapy versus chemotherapy alone for treatment of HER2-positive advanced gastric or gastro-oesophageal junction cancer (ToGA): A phase 3 , open-label, randomised controlled trial. Lancet 376: 687-697, 2010.

6. Livshits Z, Rao RB and Smith SW: An approach to chemotherapy-associated toxicity. Emerg Med Clin North Am 32: 167-203, 2014.

7. Mangia A, Caldarola L, Dell'Endice S, Scarpi E, Saragoni L, Monti M, Santini D, Brunetti O, Simone G and Silvestris N: The potential predictive role of nuclear NHERF1 expression in advanced gastric cancer patients treated with epirubicin/oxaliplatin/capecitabine first line chemotherapy. Cancer Biol Ther 16: 1140-1147, 2015

8. Tebbutt NC, Parry MM, Zannino D, Strickland AH, Van Hazel GA, Pavlakis N, Ganju V, Mellor D, Dobrovic A and Gebski VJ; Australasian Gastro-Intestinal Trials Group (AGITG): Docetaxel plus cetuximab as second-line treatment for docetaxel-refractory oesophagogastric cancer: The AGITG ATTAX2 trial. Br J Cancer 108: 771-774, 2013. 
9. Zhang ZD, Kong Y, Yang W, Zhang B, Zhang YL, Ma EM, Liu HX, Chen XB and Hua YW: Clinical evaluation of cetuximab combined with an S-1 and oxaliplatin regimen for Chinese patients with advanced gastric cancer. World J Surg Oncol 12: 115, 2014.

10. Lordick F, Kang YK, Chung HC, Salman P, Oh SC, Bodoky G, Kurteva G, Volovat C, Moiseyenko VM, Gorbunova V, et al: Capecitabine and cisplatin with or without cetuximab for patients with previously untreated advanced gastric cancer (EXPAND): A randomised, open-label phase 3 trial. Lancet Oncol 14: 490-499, 2013.

11. Waddell T, Chau I, Cunningham D, Gonzalez D, Okines AF, Okines C, Wotherspoon A, Saffery C, Middleton G Wadsley J, et al: Epirubicin, oxaliplatin, and capecitabine with or without panitumumab for patients with previously untreated advanced oesophagogastric cancer (REAL3): A randomised, open-label phase 3 trial. Lancet Oncol 14: 481-489, 2013.

12. Hotz B, Keilholz U, Fusi A, Buhr HJ and Hotz HG: In vitro and in vivo antitumor activity of cetuximab in human gastric cancer cell lines in relation to epidermal growth factor receptor (EGFR) expression and mutational phenotype. Gastric Cancer 15 : 252-264, 2012

13. Moehler M, Mueller A, Trarbach T, Lordick F, Seufferlein T, Kubicka S, Geissler M, Schwarz S, Galle PR and Kanzler S; German Arbeitsgemeinschaft Internistische Onkologie: Cetuximab with irinotecan, folinic acid and 5-fluorouracil as first-line treatment in advanced gastroesophageal cancer: A prospective multi-center biomarker-oriented phase II study. Ann Oncol 22: 1358-1366, 2011

14. Choi AH, Kim J and Chao J: Perioperative chemotherapy for resectable gastric cancer: MAGIC and beyond. World J Gastroenterol 21: 7343-7348, 2015.

15. Pietrantonio F, De Braud F, Da Prat V, Perrone F, Pierotti MA Gariboldi M, Fanetti G, Biondani P, Pellegrinelli A, Bossi I and Di Bartolomeo M: A review on biomarkers for prediction of treatment outcome in gastric cancer. Anticancer Res 33 $1257-1266,2013$

16. Gomez-Martin C, Plaza JC, Pazo-Cid R, Salud A, Pons F, Fonseca P, Leon A, Alsina M, Visa L, Rivera F, et al: Level of HER2 gene amplification predicts response and overall survival in HER2-positive advanced gastric cancer treated with trastuzumab. J Clin Oncol 31: 4445-4452, 2013.

17. Ock CY, Lee KW, Kim JW, Kim JS, Kim TY, Lee KH, Han SW, Im SA, Kim TY, Kim WH, et al: Optimal patient selection for trastuzumab treatment in HER2-positive advanced gastric cancer. Clin Cancer Res 21: 2520-2529, 2015.

18. Lee JY, Hong M, Kim ST, Park SH, Kang WK, Kim KM and Lee J: The impact of concomitant genomic alterations on treatment outcome for trastuzumab therapy in HER2-positive gastric cancer. Sci Rep 5: 9289, 2015.

19. Oyama K, Fushida S, Tsukada T, Kinoshita J, Watanabe T, Shoji M, Nakanuma S, Okamoto K, Sakai S, Makino I, et al: Evaluation of serum HER2-ECD levels in patients with gastric cancer. J Gastroenterol 50: 41-45, 2015.

20. de Azambuja E, Holmes AP, Piccart-Gebhart M, Holmes E, Di Cosimo S, Swaby RF, Untch M, Jackisch C, Lang I, Smith I, et al: Lapatinib with trastuzumab for HER2-positive early breast cancer (NeoALTTO): Survival outcomes of a randomised, open-label, multicentre, phase 3 trial and their association with pathological complete response. Lancet Oncol 15: 1137-1146, 2014.

21. Hecht JR, Bang YJ, Qin SK, Chung HC, Xu JM, Park JO, Jeziorski K, Shparyk Y, Hoff PM, Sobrero A, et al: Lapatinib in combination with capecitabine plus oxaliplatin in human epidermal growth factor receptor 2-positive advanced or metastatic gastric, esophageal, or gastroesophageal adenocarcinoma: TRIO-013/LOGiC-A randomized phase III trial. J Clin Oncol 34: 443-451, 2016.

22. Satoh T, Xu RH, Chung HC, Sun GP, Doi T, Xu JM, Tsuji A, Omuro Y, Li J, Wang JW, et al: Lapatinib plus paclitaxel versus paclitaxel alone in the second-line treatment of HER2-amplified advanced gastric cancer in Asian populations: TyTAN-a randomized, phase III study. J Clin Oncol 32: 2039-2049, 2014.

23. Oshima Y, Tanaka H, Murakami H, Ito Y, Furuya T, Kondo E, Kodera $\mathrm{Y}$ and Nakanishi H: Lapatinib sensitivities of two novel trastuzumab-resistant HER2 gene-amplified gastric cancer cell lines. Gastric Cancer 17: 450-462, 2014.

24. Eto K, Iwatsuki M, Watanabe M, Ida S, Ishimoto T, Iwagami S, Baba Y, Sakamoto Y, Miyamoto Y, Yoshida N and Baba H: The microRNA-21/PTEN pathway regulates the sensitivity of HER2-positive gastric cancer cells to trastuzumab. Ann Surg Oncol 21: 343-350, 2014.
25. Zhang X, Park JS, Park KH, Kim KH, Jung M, Chung HC, Rha SY and Kim HS: PTEN deficiency as a predictive biomarker of resistance to HER2-targeted therapy in advanced gastric cancer. Oncology 88: 76-85, 2015.

26. Hong YS, Kim J, Pectasides E, Fox C, Hong SW, Ma Q, Wong GS, Peng S, Stachler MD, Thorner AR, et al: Src mutation induces acquired lapatinib resistance in ERBB2-amplified human gastroesophageal adenocarcinoma models. PLoS One 9: e109440, 2014

27. Sanford M: Trastuzumab: A review of its use in HER2-positive advanced gastric cancer. Drugs 73: 1605-1615, 2013.

28. Gomez-Martin C, Lopez-Rios F, Aparicio J, Barriuso J, García-Carbonero R, Pazo R, Rivera F, Salgado M, Salud A, Vázquez-Sequeiros E and Lordick F: A critical review of HER2-positive gastric cancer evaluation and treatment: From trastuzumab, and beyond. Cancer Lett 351: 30-40, 2014

29. Sanford M: Subcutaneous trastuzumab: A review of its use in HER2-positive breast cancer. Target Oncol 9: 85-94, 2014.

30. Kim HP, Han SW, Song SH, Jeong EG, Lee MY, Hwang D, Im SA, Bang YJ and Kim TY: Testican-1-mediated epithelial-mesenchymal transition signaling confers acquired resistance to lapatinib in HER2-positive gastric cancer. Oncogene 33: 3334-3341, 2014

31. De Silva N, Schulz L, Paterson A, Qain W, Secrier M, Godfrey E, Cheow H, O'Donovan M, Lao-Sirieix P, Jobanputra M, et al: Molecular effects of Lapatinib in the treatment of HER2 overexpressing oesophago-gastric adenocarcinoma. Br J Cancer 113: $1305-1312,2015$

32. Abdel-Rahman O: Targeting vascular endothelial growth factor (VEGF) pathway in gastric cancer: Preclinical and clinical aspects. Crit Rev Oncol Hematol 93: 18-27, 2015.

33. Escudier B, Pluzanska A, Koralewski P, Ravaud A, Bracarda S, Szczylik C, Chevreau C, Filipek M, Melichar B, Bajetta E, et al: Bevacizumab plus interferon alfa-2a for treatment of metastatic renal cell carcinoma: A randomised, double-blind phase III trial. Lancet 370: 2103-2111, 2007.

34. Qu CY, Zheng Y, Zhou M, Zhang Y, Shen F, Cao J and Xu LM: Value of bevacizumab in treatment of colorectal cancer: A meta-analysis. World J Gastroenterol 21: 5072-5080, 2015.

35. Lauro S, Onesti CE, Righini R and Marchetti P: The use of bevacizumab in non-small cell lung cancer: An update. Anticancer Res 34: 1537-1545, 2014.

36. Ohtsu A, Shah MA, Van Cutsem E, Rha SY, Sawaki A, Park SR, Lim HY, Yamada Y, Wu J, Langer B, et al: Bevacizumab in combination with chemotherapy as first-line therapy in advanced gastric cancer: A randomized, double-blind, placebo-controlled phase III study. J Clin Oncol 29: 3968-3976, 2011.

37. Shen L, Li J, Xu J, Pan H, Dai G, Qin S, Wang L, Wang J, Yang Z, Shu Y, et al: Bevacizumab plus capecitabine and cisplatin in Chinese patients with inoperable locally advanced or metastatic gastric or gastroesophageal junction cancer: Randomized, double-blind, phase III study (AVATAR study). Gastric Cancer 18: 168-176, 2015.

38. Yamashita-Kashima Y, Fujimoto-Ouchi K, Yorozu K, Kurasawa M, Yanagisawa M, Yasuno H and Mori K: Biomarkers for antitumor activity of bevacizumab in gastric cancer models. BMC Cancer 12: 37, 2012.

39. Van Cutsem E, de Haas S, Kang YK, Ohtsu A, Tebbutt NC, Ming Xu J, Peng Yong W, Langer B, Delmar P, Scherer SJ and Shah MA: Bevacizumab in combination with chemotherapy as first-line therapy in advanced gastric cancer: A biomarker evaluation from the AVAGAST randomized phase III trial. J Clin Oncol 30: 2119-2127, 2012.

40. Han K, Jin J, Maia M, Lowe J, Sersch MA and Allison DE: Lower exposure and faster clearance of bevacizumab in gastric cancer and the impact of patient variables: Analysis of individual data from AVAGAST phase III trial. AAPS J 16: 1056-1063, 2014

41. Hacker UT, Escalona-Espinosa L, Consalvo N, Goede V, Schiffmann L, Scherer SJ, Hedge P, Van Cutsem E, Coutelle O and Büning $\mathrm{H}$ : Evaluation of Angiopoietin-2 as a biomarker in gastric cancer: Results from the randomised phase III AVAGAST trial. Br J Cancer 114: 855-862, 2016.

42. Poole RM and Vaidya A: Ramucirumab: First global approval. Drugs 74: 1047-1058, 2014

43. Wilke H, Muro K, Van Cutsem E, Oh SC, Bodoky G, Shimada Y, Hironaka S, Sugimoto N, Lipatov O, Kim TY, etal: Ramucirumab plus paclitaxel versus placebo plus paclitaxel in patients with previously treated advanced gastric or gastro-oesophageal junction adenocarcinoma (RAINBOW): A double-blind, randomised phase 3 trial. Lancet Oncol 15: 1224-1235, 2014. 
44. Fuchs CS, Tomasek J, Yong CJ, Dumitru F, Passalacqua R, Goswami C, Safran H, Dos Santos LV, Aprile G, Ferry DR, et al: Ramucirumab monotherapy for previously treated advanced gastric or gastro-oesophageal junction adenocarcinoma (REGARD): An international, randomised, multicentre, placebo-controlled, phase 3 trial. Lancet 383: 31-39, 2014.

45. Iacovelli R, Pietrantonio F, Farcomeni A, Maggi C, Palazzo A, Ricchini F, de Braud F and Di Bartolomeo M: Chemotherapy or targeted therapy as second-line treatment of advanced gastric cancer. A systematic review and meta-analysis of published studies. PLoS One 9: e108940, 2014

46. Li J, Qin S, Xu J, Xiong J, Wu C, Bai Y, Liu W, Tong J, Liu Y, $\mathrm{Xu} \mathrm{R}$, et al: Randomized, double-blind, placebo-controlled phase III trial of apatinib in patients with chemotherapy-refractory advanced or metastatic adenocarcinoma of the stomach or gastroesophageal junction. J Clin Oncol 34: 1448-1454, 2016.

47. Fioroni I, Dell'Aquila E, Pantano F, Intagliata S, Caricato M, Vincenzi B, Coppola R, Santini D and Tonini G: Role of c-mesenchymal-epithelial transition pathway in gastric cancer. Expert Opin Pharmacother 16: 1195-1207, 2015.

48. Zhang Y, Doshi S and Zhu M: Pharmacokinetics and pharmacodynamics of rilotumumab: A decade of experience in preclinical and clinical cancer research. Br J Clin Pharmacol 80: 957-964, 2015.

49. Iveson T, Donehower RC, Davidenko I, Tjulandin S, Deptala A, Harrison M, Nirni S, Lakshmaiah K, Thomas A, Jiang Y, et al: Rilotumumab in combination with epirubicin, cisplatin, and capecitabine as first-line treatment for gastric or oesophagogastric junction adenocarcinoma: An open-label, dose de-escalation phase $1 \mathrm{~b}$ study and a double-blind, randomised phase 2 study. Lancet Oncol 15: 1007-1018, 2014.

50. Catenacci DVT, Tebbutt NC, Davidenko I, Murad AM, Al-Batran SE, Ilson DH, Tjulandin S, Gotovkin E, Karaszewska B, Bondarenko I, et al: Rilotumumab plus epirubicin, cisplatin, and capecitabine as first-line therapy in advanced MET-positive gastric or gastro-oesophageal junction cancer (RILOMET-1) A randomised, double-blind, placebo-controlled, phase 3 trial. Lancet Oncol 18: 1467-1482, 2017.

51. Shah MA, Bang YJ, Lordick F, Alsina M, Chen M, Hack SP, Bruey JM, Smith D, McCaffery I, Shames DS, et al: Effect of fluorouracil, leucovorin, and oxaliplatin with or without onartuzumab in HER2-negative, MET-positive gastroesophageal adenocarcinoma: The METGastric randomized clinical trial. JAMA Oncol 3: 620-627, 2017.

52. Shah MA, Wainberg ZA, Catenacci DV, Hochster HS, Ford J, Kunz P, Lee FC, Kallender H, Cecchi F, Rabe DC, et al: Phase II study evaluating 2 dosing schedules of oral foretinib (GSK1363089), cMET/VEGFR2 inhibitor, in patients with metastatic gastric cancer. PLoS One 8: e54014, 2013.

53. Wu Z, Zhang Z, Ge X, Lin Y, Dai C, Chang J, Liu X, Geng R, Wang $\mathrm{C}$, Chen $\mathrm{H}$, et al: Identification of short-form RON as a novel intrinsic resistance mechanism for anti-MET therapy in MET-positive gastric cancer. Oncotarget 6: 40519-40534, 2015.

54. Kwak EL, Ahronian LG, Siravegna G, Mussolin B, Godfrey JT, Clark JW, Blaszkowsky LS, Ryan DP, Lennerz JK, Iafrate AJ, et al: Molecular heterogeneity and receptor coamplification drive resistance to targeted therapy in MET-amplified esophagogastric cancer. Cancer Discov 5: 1271-1281, 2015.

55. Ji F, Liu X, Wu Y, Fang X and Huang G: Overexpression of PI3K p110 $\alpha$ contributes to acquired resistance to MET inhibitor in MET-amplified SNU-5 gastric xenografts. Drug Des Devel Ther 9: 5697-5704, 2015.

56. Musiani D, Konda JD, Pavan S, Torchiaro E, Sassi F, Noghero A, Erriquez J, Perera T, Olivero M and Di Renzo MF: Heat-shock protein 27 (HSP27, HSPB1) is up-regulated by MET kinase inhibitors and confers resistance to MET-targeted therapy. FASEB J 28: 4055-4067, 2014

57. Chen CT, Kim H, Liska D, Gao S, Christensen JG and Weiser MR: MET activation mediates resistance to lapatinib inhibition of HER2-amplified gastric cancer cells. Mol Cancer Ther 11: 660-669, 2012

58. Sukawa Y, Yamamoto H, Nosho K, Ito M, Igarashi H, Naito T, Mitsuhashi K, Matsunaga Y, Takahashi T, Mikami M, et al: HER2 expression and PI3K-Akt pathway alterations in gastric cancer. Digestion 89: 12-17, 2014.
59. An X, Wang F, Shao Q, Wang FH, Wang ZQ, Wang ZQ, Chen C, $\mathrm{Li} \mathrm{C}$, Luo HY, Zhang DS, et al: MET amplification is not rare and predicts unfavorable clinical outcomes in patients with recurrent/metastatic gastric cancer after chemotherapy. Cancer 120 675-682, 2014

60. Xu K, Liu P and Wei W: mTOR signaling in tumorigenesis. Biochim Biophys Acta 1846: 638-654, 2014.

61. Hasskarl J: Everolimus. Recent Results Cancer Res 201: 373-392, 2014.

62. Ohtsu A, Ajani JA, Bai YX, Bang YJ, Chung HC, Pan HM, Sahmoud T, Shen L, Yeh KH, Chin K, et al: Everolimus for previously treated advanced gastric cancer: Results of the randomized, double-blind, phase III GRANITE-1 study. J Clin Oncol 31: 3935-3943, 2013 .

63. Wainberg ZA, Soares HP, Patel R, DiCarlo B, Park DJ, Liem A, Wang HJ, Yonemoto L, Martinez D, Laux I, et al: Phase II trial of everolimus in patients with refractory metastatic adenocarcinoma of the esophagus, gastroesophageal junction and stomach: Possible role for predictive biomarkers. Cancer Chemother Pharmacol 76: 61-67, 2015.

64. Zhu Y, Tian T, Zou J, Wang Q, Li Z, Li Y, Liu X, Dong B, Li N, Gao J and Shen L: Dual PI3K/mTOR inhibitor BEZ235 exerts extensive antitumor activity in HER2-positive gastric cancer. BMC Cancer 15: 894, 2015

65. Fuereder T, Jaeger-Lansky A, Hoeflmayer D, Preusser M, Strommer S, Cejka D, Koehrer S, Crevenna R and Wacheck V: mTOR inhibition by everolimus counteracts VEGF induction by sunitinib and improves anti-tumor activity against gastric cancer in vivo. Cancer Lett 296: 249-256, 2010.

66. Jaeger-Lansky A, Cejka D, Ying L, Preusser M, Hoeflmayer D, Fuereder T, Koehrer S and Wacheck V: Effects of vatalanib on tumor growth can be potentiated by mTOR blockade in vivo. Cancer Biol Ther 9: 919-927, 2010.

67. Keir ME, Butte MJ, Freeman GJ and Sharpe AH: PD-1 and its ligands in tolerance and immunity. Annu Rev Immunol 26: 677-704, 2008.

68. Freeman GJ, Long AJ, Iwai Y, Bourque K, Chernova T, Nishimura H, Fitz LJ, Malenkovich N, Okazaki $T$, Byrne MC, et al: Engagement of the PD-1 immunoinhibitory receptor by a novel $\mathrm{B} 7$ family member leads to negative regulation of lymphocyte activation. J Exp Med 192: 1027-1034, 2000.

69. Muro K, Chung HC, Shankaran V, Geva R, Catenacci D, Gupta S, Eder JP, Golan T, Le DT, Burtness B, et al: Pembrolizumab for patients with PD-L1-positive advanced gastric cancer (KEYNOTE-012): A multicentre, open-label, phase 1b trial. Lancet Oncol 17: 717-726, 2016.

70. Fuchs CS, Doi T, Jang RW, Muro K, Satoh T, Machado M, Sun W, Jalal SI, Shah MA, Metges JP, et al: Safety and efficacy of pembrolizumab monotherapy in patients with previously treated advanced gastric and gastroesophageal junction cancer: Phase 2 clinical KEYNOTE-059 trial. JAMA Oncol: Mar 15, 2018 (Epub ahead of print).

71. Ohtsu A, Tabernero J, Bang YJ, Fuchs CS, Sun L, Wang Z, Csiki I, Koshiji and Cutsem EV: Pembrolizumab versus paclitaxel as second-line therapy for advanced gastric or gastroesophageal junction (GEJ) adenocarcinoma: Phase 3 KEYNOTE-061 study. J Clin Oncol 34 (15 Suppl): TPS4137, 2016.

72. Tabernero J, Bang YJ, Fuchs CS, Ohtsu A, Kher U, Lam B, Koshiji M and Cutsem V: KEYNOTE-062: Phase III study of pembrolizumab alone or in combination with chemotherapy versus chemotherapy alone as first-line therapy for advanced gastric or gastroesophageal junction (GEJ) adenocarcinoma. J Clin Oncol 34 (4 Suppl): TPS185, 2016.

73. Camacho LH: CTLA-4 blockade with ipilimumab: Biology, safety, efficacy, and future considerations. Cancer Med 4: 661-672, 2015.

74. Moehler MH, Janjigian YY, Adenis A, Aucoin JS, Boku N, Chau I, Cleary JM, Feeney KT, Franke FA, Mendez GA, et al: CheckMate 649: A randomized, multicenter, open-label, phase 3 study of nivolumab (nivo) + ipilimumab (ipi) or nivo + chemotherapy (CTX) vs CTX alone in pts with previously untreated advanced (adv) gastric $(\mathrm{G})$ or gastroesophageal junction (GEJ) cancer. J Clin Oncol 35 (15 Suppl): TPS4132, 2017. 